\title{
Genomic profiles of a hepatoblastoma from a patient with Beckwith-Wiedemann syndrome with uniparental disomy on chromosome 11p15 and germline mutation of APC and PALB2
}

\author{
Shinn Young Kim ${ }^{1,4,5,6}$, Seung-Hyun Jung ${ }^{1,2}$, Min Sung Kim²,3, Mi-Ryung Han ${ }^{1,4}$, \\ Hyeon-Chun Park ${ }^{1,4}$, Eun Sun Jung7, Sung Hak Lee ${ }^{7}$, Sug Hyung Lee ${ }^{1,2,3}$ and Yeun- \\ Jun Chung ${ }^{1,4,5}$ \\ ${ }^{1}$ Department of Precision Medicine Research Center, College of Medicine, The Catholic University of Korea, Seoul, South Korea \\ ${ }^{2}$ Department of Cancer Evolution Research Center, College of Medicine, The Catholic University of Korea, Seoul, South Korea \\ ${ }^{3}$ Department of Pathology, College of Medicine, The Catholic University of Korea, Seoul, South Korea \\ ${ }^{4}$ Department of Integrated Research Center for Genome Polymorphism, College of Medicine, The Catholic University of \\ Korea, Seoul, South Korea \\ ${ }^{5}$ Department of Microbiology, College of Medicine, The Catholic University of Korea, Seoul, South Korea \\ ${ }^{6}$ Department of Surgery, College of Medicine, The Catholic University of Korea, Seoul, South Korea \\ ${ }^{7}$ Department of Hospital Pathology, College of Medicine, The Catholic University of Korea, Seoul, South Korea \\ Correspondence to: Yeun-Jun Chung, email: yejun@catholic.ac.kr \\ Sug Hyung Lee, email: suhulee@catholic.ac.kr \\ Keywords: hepatoblastoma, uniparental disomy, mutation, copy number alteration, BWS \\ Received: May 30, $2017 \quad$ Accepted: August 07, $2017 \quad$ Published: August 24, 2017 \\ Copyright: Kim et al. This is an open-access article distributed under the terms of the Creative Commons Attribution License 3.0 \\ (CC BY 3.0), which permits unrestricted use, distribution, and reproduction in any medium, provided the original author and source \\ are credited.
}

\section{ABSTRACT}

Beckwith-Wiedemann syndrome (BWS) is a congenital overgrowth disorder mainly associated with altered genomic imprinting at chromosome 11p15.5. Children with BWS, especially uniparental disomy (UPD) at 11p15.5, are at increased risk of embryonal tumors including hepatoblastoma. Although genetic alterations of sporadic hepatoblastomas have been identified, integrated germline and somatic alterations of BWS-related hepatoblastoma have not been reported. For this, we performed wholeexome sequencing and genome-wide loss of heterozygosity/copy number analyses using a single nucleotide polymorphism (SNP) array for a hepatoblastoma in a BWS infant with paternal UPD at chromosome 11p15.5. We found germline 11p15.5 UPD as well as germline mutations of APC and PALB2 in the patient. At the somatic level, we found a CTNNB1 hotspot mutation and chromosome 1q gain in the tumor. The development of hepatoblastoma in this case might be explained by predisposition of the germline events (11p15.5 UPD, mutations of APC and PALB2) and later by somatic events with CTNNB1 somatic mutation and 1q gain. To our knowledge, this is the first report of germline and somatic genomic alteration profiles in hepatoblastoma arising from BWS. Clinically, our results provide a rationale for performing a more strict and intense protocol for hepatoblastoma surveillance in a high-risk BWS infant, such as the UPD-carrying case, for early detection and treatment.

\section{INTRODUCTION}

Beckwith-Wiedemann syndrome (BWS, OMIM $130650)$, either sporadic $(>85 \%)$ or familial $(<15 \%)$, is caused by mutation or deletion of imprinted genes CDKN1C, H19 and LITI as well as by hypermethylation in the H19/IGF2-imprinting control region within the chromosome 11 p15.5 region [1]. Some other sporadic 
BWSs harbor paternal uniparental disomy (UPD) that results in the replacement of the maternal $11 \mathrm{p} 15.5$ with an extra paternal copy. About $20 \%$ of BWS patients have paternal UPD [2]. UPD occurs in BWS as a postfertilization mitotic recombination event that results in somatic mosaicism [3]. Patients with BWS are characterized by phenotypic presentations of overgrowth including macrosomia, macroglossia, ear defects and anterior abdominal wall defects as well as severe hypoglycemia [2].

The incidence of tumors in BWS patients is estimated to be $7.5 \%$ (range $4-21 \%$ ), which is far higher (relative risk of 676) than that in other children [4]. Such tumors include Wilms' tumor (43\%), hepatoblastoma (20\%) and adrenocortical carcinoma (7\%), and usually occur before 4 years of age $(\sim 90 \%)$ [5]. BWS is caused by 11 p15.5 alterations that may lead to tissue overgrowth for the phenotypic presentations and may also provide genetic backgrounds for tumor development. However, because most BWS patients with the $11 \mathrm{p} 15.5$ alterations do not develop tumors, it is possible that there might be other genetic factors that predispose to tumor development. Hepatoblastoma accounts for approximately $1 \%$ of childhood tumors but is the most common primary tumor in childhood liver [6]. It sometimes develops in patients with familial diseases including familial adenomatous polyposis (FAP) and BWS, but usually occurs as sporadic cases [7]. In sporadic hepatoblastomas even without FAP manifestations, de novo germline $A P C$ mutations are found [8].

Somatic mutations are crucial in the development of both hereditary and sporadic tumors. Recent whole-exome sequencing (WES)-based mutation studies identified high frequencies of somatic mutations of $A P C, \beta$-catenin (CTNNB1: around 80\%) and NFE2L2 (10\%), as well as germline $A P C$ mutations $(60 \%)$ in hepatoblastomas [9-11]. To our knowledge, only one case of hepatoblastoma in a BWS patient (11p15.5 alteration type was not available) has been studied by WES [9]. This analysis revealed a somatic CTNNB1 mutation, but no germline $A P C$ mutation.

To further extend the knowledge on BWS-associated hepatoblastoma development, we performed WES of a hepatoblastoma in a BWS infant with paternal UPD on chromosome $11 \mathrm{p} 15.5$ and germline $A P C$ mutation in this study.

\section{RESULTS}

\section{Clinical feature of the patient}

An infant boy was born by caesarian section at gestational age of 38 weeks due to his intrauterine overgrowth. Apgar score was 6 at 1 minute and 8 at 5 minutes. He had macroglossia and macrosomia. His weight was $4.825 \mathrm{~kg}$ ( $>90$ percentile), height was 53 $\mathrm{cm}$ (90 percentile), and head conference was $34 \mathrm{~cm}$ (50 percentile). His initial blood sugar level was $17 \mathrm{mg} /$ dl (neonatal hypoglycemia), which was recovered with glucose injection by the third day of birth. Presence of three of the five common features associated with BWS (macroglossia, macrosomia, midline abdominal wall defects, ear creases/ear pits, and neonatal hypoglycemia) prompted the diagnosis as BWS. His parents as well as the second and third degree relatives did not have any evidence to suspect BWS. They did not have histories of FAP nor hepatoblastoma.

The baby was discharged at 1 month of age with a 3-month tumor screening schedule by abdominal ultrasonography and serum alpha-fetoprotein (AFP) as described elsewhere [12]. On his first visit to the outside clinic ( +1 week after discharge), the AFP level was 6,428 $\mathrm{ng} / \mathrm{ml}$, which was decreasing compared to the initial AFP level of $124,704 \mathrm{ng} / \mathrm{ml}$ (Supplementary Figure 1). On the second visit ( +9 weeks), it appeared that the AFP level was in the course of the natural decline and abdominal ultrasonography showed no evidence of hepatic tumor mass. On the third visit ( +17 weeks), the AFP level had slightly increased, but abdominal ultrasonography was not performed due to the parent's refusal. On the fourth visit (+29 weeks), his serum AFP level was elevated to $>200,000 \mathrm{ng} / \mathrm{ml}$ and abdominal computer tomography scan revealed a well-defined heterogeneous liver mass $(6.5 \times$ $6.2 \mathrm{~cm}$ ) (Figures 1A, 1B) suspicious of a hepatoblastoma. The hepatoblastoma was classified as PRETEXT stage II, a staging system for primary malignant liver tumors of childhood, that involved two sections (left medial and right anterior sections) of the four without any evidence of distant metastasis. He received transarterial chemoembolization with adriamycin and carboplatin, and neoadjuvant chemotherapy with cisplatin, vincristine and 5-fluorouracil. After 3 cycles of chemotherapy, hepatic segmentectomy (segment I, IV) was performed for curative surgery. The histopathologic diagnosis was hepatoblastoma with epithelial type (Figure 1C, 1D). He is now at complete remission state at 3 years post-operatively with five subsequent cycles of chemotherapy performed.

\section{Molecular-genetic basis of BWS diagnosis}

OncoScan SNP array analysis was performed to help the genetic diagnosis and molecular characterization of the BWS in this patient. Whole genome view with B-allele frequency for both normal (Figure 2A) and hepatoblastoma tumor tissue (Figure 2B) revealed copy-neutral LOH at chromosome 11p15.5 (chr11:192,764-45,663,568), indicating a germline UPD as a molecular subtype of BWS. When we estimated the UPD levels based on their B-allele frequencies, tumor tissue showed slightly higher UPD fraction $(60 \%)$ than normal tissue $(50 \%)$. There was a somatic copy number alteration (CNA) in the tumor genome (1q gain), while other chromosomes were largely 
stable (Figure 2B). The 1q gain has previously reported as the most frequent CNA in hepatoblastomas [11].

\section{Catalogue of somatic mutations}

Mean coverage of the WES depth was $101 \mathrm{X}$ for tumor genome and $54 \mathrm{X}$ for normal, with an average of $93 \%$ of bases covered by at least 10 reads. After calling somatic mutations using MuTect and SomaticIndelDetector with default parameters, reads less than 20 were filtered out. After the filtering process, a total of 15 somatic mutations (12 point mutations and three indels) were called (Supplementary Table 1), corresponding to 0.5 somatic mutations per megabase
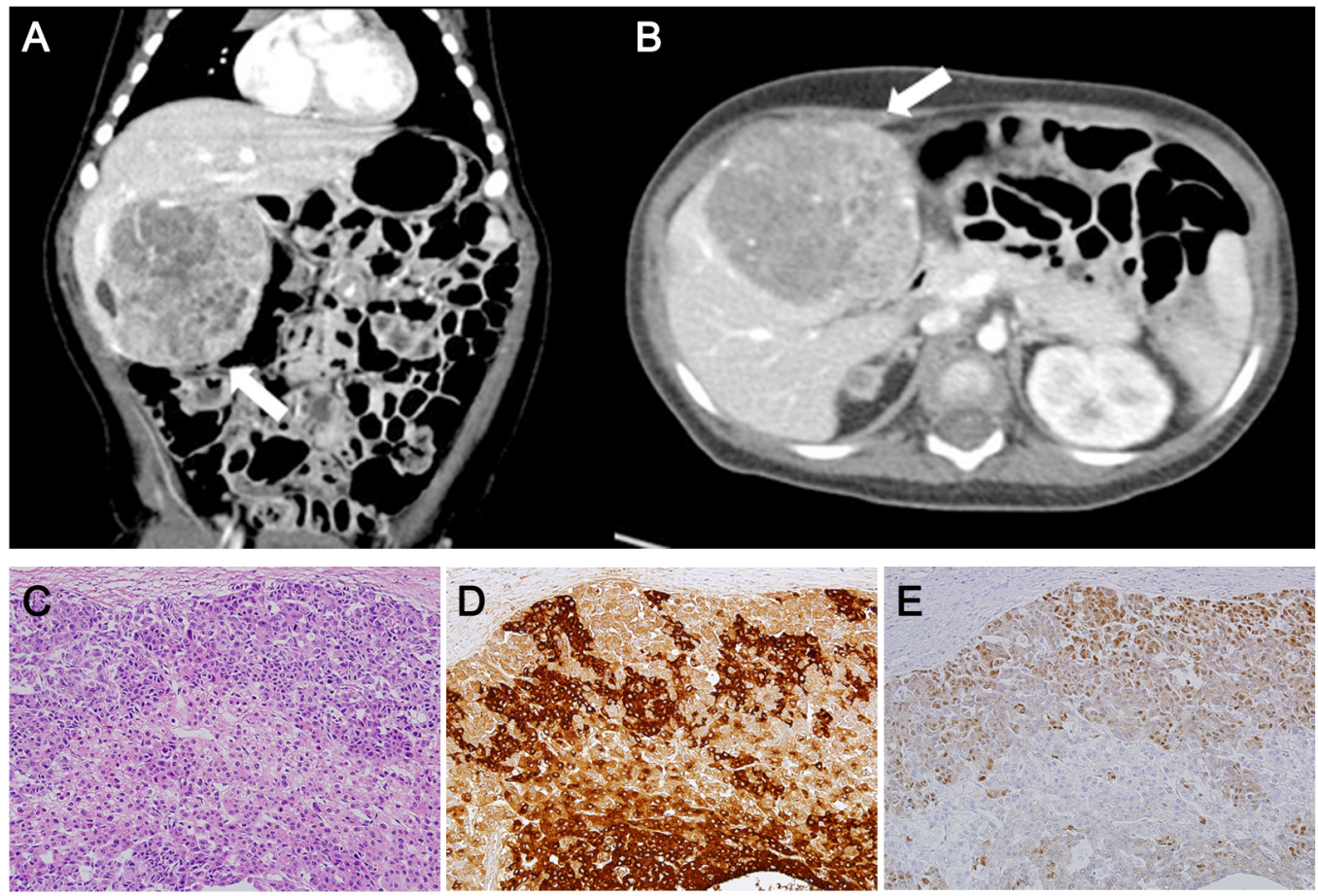

Figure 1: Abdominal computer tomography and histopathology of the case. Abdominal computer tomography scan at 8 months (coronary (A) and axial (B) view) showing a huge and well-defined heterogeneous mass suspicious of hepatoblastoma classified as PRETEXT stage II (arrow). Hematoxylin and Eosin staining (C), Immunohistochemistry for alpha fetoprotein (D) and $\beta$-catenin (E) show compatible features of hepatoblastoma.

A
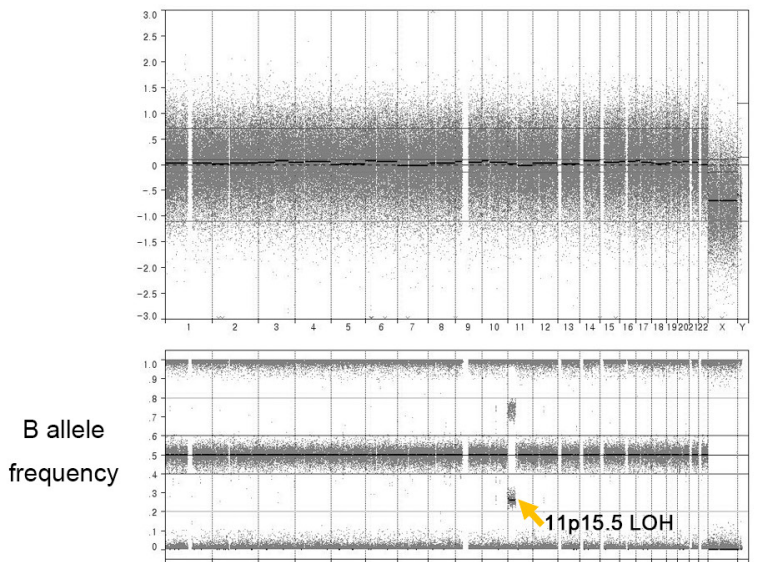

B
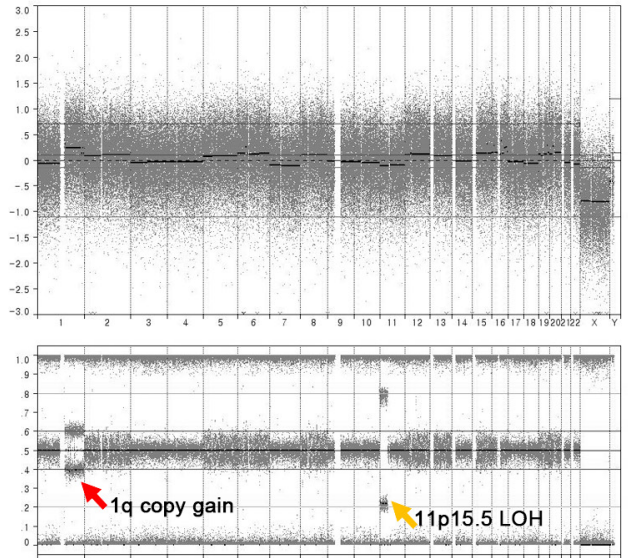

Figure 2: SNP array analysis of both normal (A) and tumor (B) genomes. Both samples show copy-neutral loss of heterozygosity at chromosome 11p15 (chr11:192,764-45,663,568) which suggests germline uniparental disomy as the genetic subtype of BWS (orange arrow). There is a somatic copy gain at chromosome 1q of the tumor genome (red arrow). 
$(\mathrm{Mb})$, which was similar to those of previously reported for hepatoblastoma $(0.067$ per $\mathrm{Mb})[10,11]$ and TLCT (0.73 per $\mathrm{Mb}$ ) [10], but much lower than that of adult $\mathrm{HCC}$ (TCGA provisional data, 2.73 per $\mathrm{Mb}$ ) (Figure 3A). C:G to $\mathrm{T}$ :A transitions were predominant, consistent with the mutation spectra of liver cancers in adults and children $[10,13]$ (Figure 3B).

Twelve non-silent gene mutations were detected: CTNNB1, OVGP1, SCN2A, HCLS1, MUC4, MUC6 OR10S1, TNRC6A, SLC9A5, SUGP2, ZNF99, and ZNF606 (Supplementary Table 1). Of them, only CTNNB1 was catalogued in the COSMIC cancer Gene Census, suggesting that the $C T N N B 1$ mutation might be a driver. CTNNB1 c.G101T (p.G34V) somatic mutation was further confirmed with Sanger sequencing (Figure 4A). The CTNNB1 p.G34V has been reported as a recurrent driver mutation occurring in hepatoblastoma [14] and in other human cancers [15]. Immunohistochemistry revealed $\beta$-catenin nuclear staining in hepatoblastoma cells, suggesting activated Wnt signaling in the tumor (Figure 1E). Functional annotation of the mutated genes using the DAVID database [16] revealed that the Wnt signaling pathway was the most associated biological process (Supplementary Table 2), indicating that the activation of the Wnt pathway may be the key driver in sporadic hepatoblastoma [9-11] and in BWS-related hepatoblastoma.

\section{Mutation signatures}

Mutational signature analyses showed that the hepatoblastoma with BWS in our study was dominated
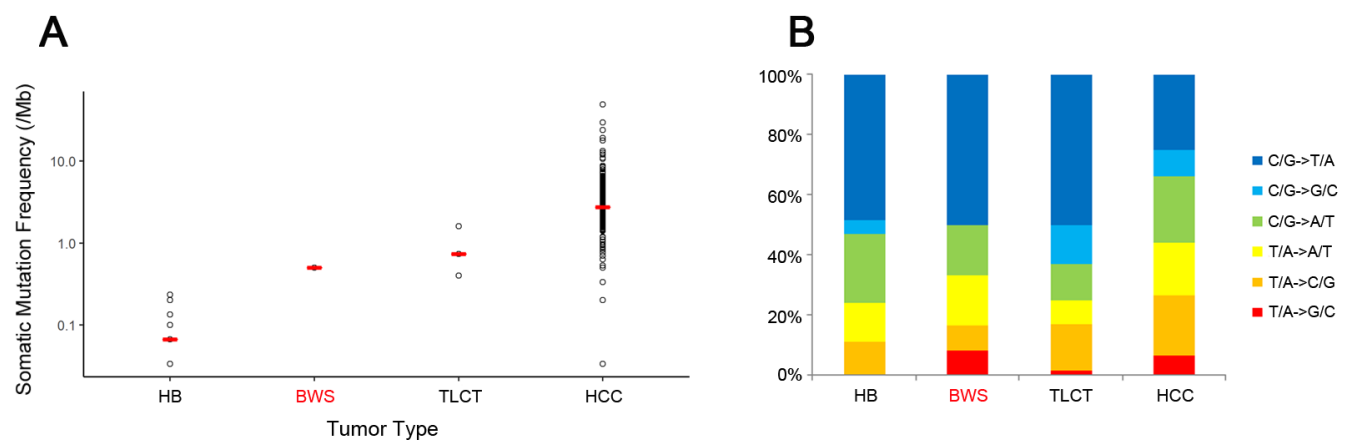

Figure 3: Comparison of somatic mutation frequency (A) and mutation spectra (B) occurring in BWS and other liver related cancers. Mean values of somatic mutation frequencies are represented with red bars in plot A. Abbreviations are: HB, hepatoblastoma; BWS, Beckwith-Wiedemann syndrome; TLCT, transient liver cell tumor; HCC, hepatocellular carcinoma

A
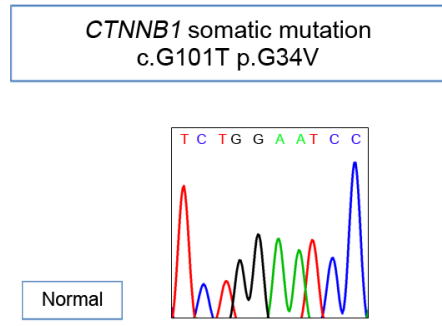

Tumor

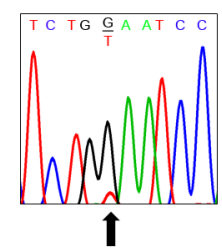

B

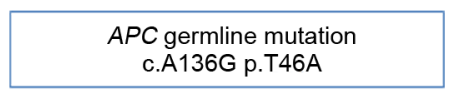

Normal
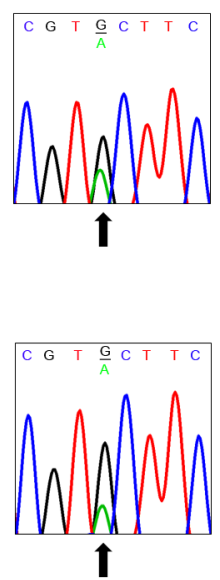

C

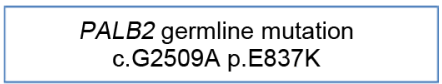

Normal

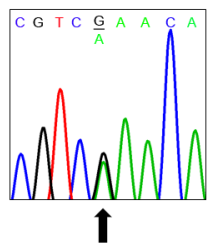

Tumor

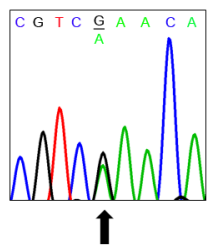

Figure 4: Sanger validation of the variants detected by whole exome sequencing. $C T N N B 1$ somatic (A), $A P C$ germline (B), and PALB2 germline (C) mutations. 
by the $\mathrm{C}>\mathrm{T}$ transition in the context of $\mathrm{GpCpG}$ trinucleotides (Figure 5A and 5B). A previously reported hepatoblastoma with BWS (Caucasian) [9] was dominated by $\mathrm{T}>\mathrm{A}$ transition in the context of $\mathrm{CpTpC}$ trinucleotides. The mutational signatures of other liver tumors (hepatoblastoma, HCC and TLCT) were dominated by $\mathrm{C}>\mathrm{T}$ transition in the context of ApCpG trinucleotides (Figure 5A and 5B). Similarities between mutational signatures were calculated using a cosine correlation similarity which ranges between 0 and 1 . Mutation signatures in sporadic hepatoblastoma exhibited the signature 1 of the 30 known signatures in the COSMIC database (cosine similarity $\geq 0.8$ ), whereas those in the two hepatoblastomas with BWS (our case and the Caucasian BWS [9]) did not show any known signature (cosine similarity $<0.8$ ) (Figure $5 \mathrm{C}$ ).

\section{Germline mutations}

We analyzed the WES data of the normal tissue genome to find any germline alterations possibly contributing to the predisposition to hepatoblastoma in BWS. Germline variants were called using the GATK HaplotypeCaller with default parameters. Synonymous variants and variants of more than $1 \%$ minor allele frequency in 1,000 Genomes Project or The Exome Aggregation Consortium (ExAC) database were excluded. Among the filtered variants listed, we searched for the genes that were catalogued in the COSMIC cancer Gene Census as known germline mutations predisposing to cancers (Supplementary Table 3) and identified a heterozygous $A P C$ missense mutation c.A136G (p.T46A), which was further validated with Sanger sequencing (Figure 4B). This mutation was identified in both normal and tumor tissues of the patient and was confirmed as a germline mutation. The parents of the BWS infant had neither family history of FAP nor clinical symptom of FAP. The APC p.T46A mutation was a novel one that had not been reported. In addition, we detected a germline mutation in PALB2 gene c.G2509A (p.E837K) encoding a protein for double strand break repair (Figure 4C) [17]. The damaging scores of the mutants $A P C$
A

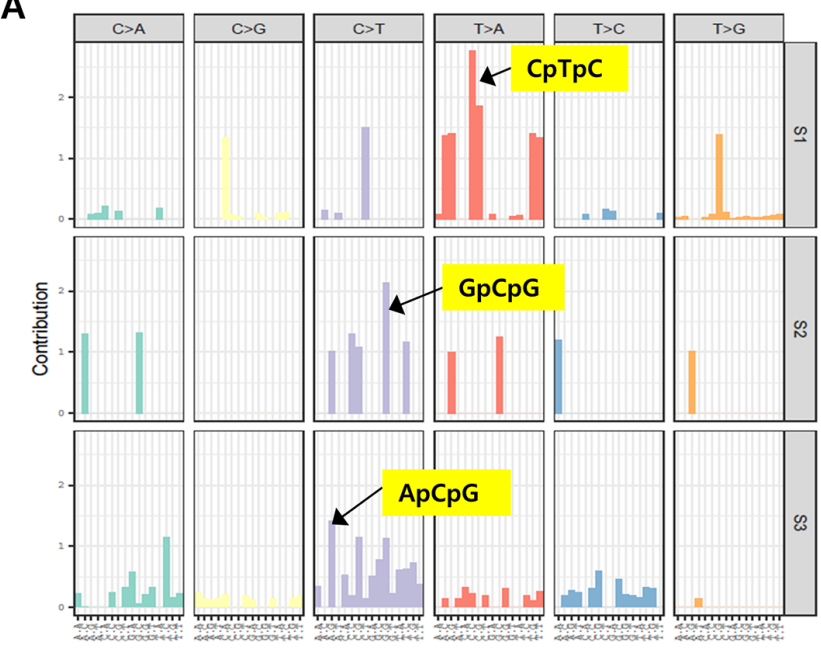

C

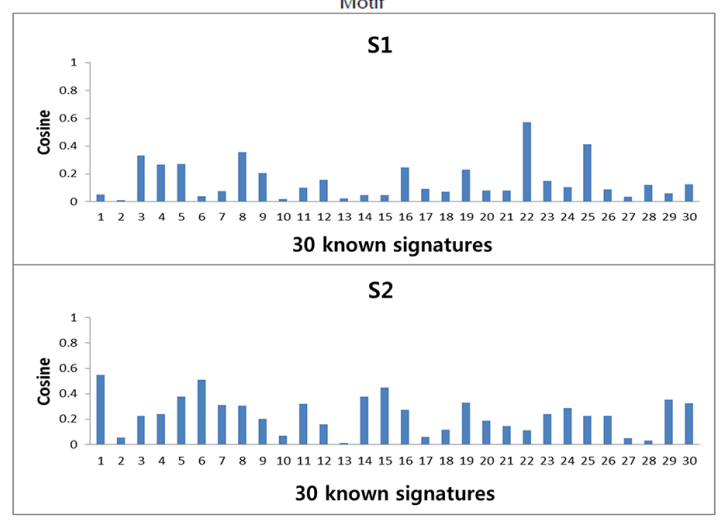

B
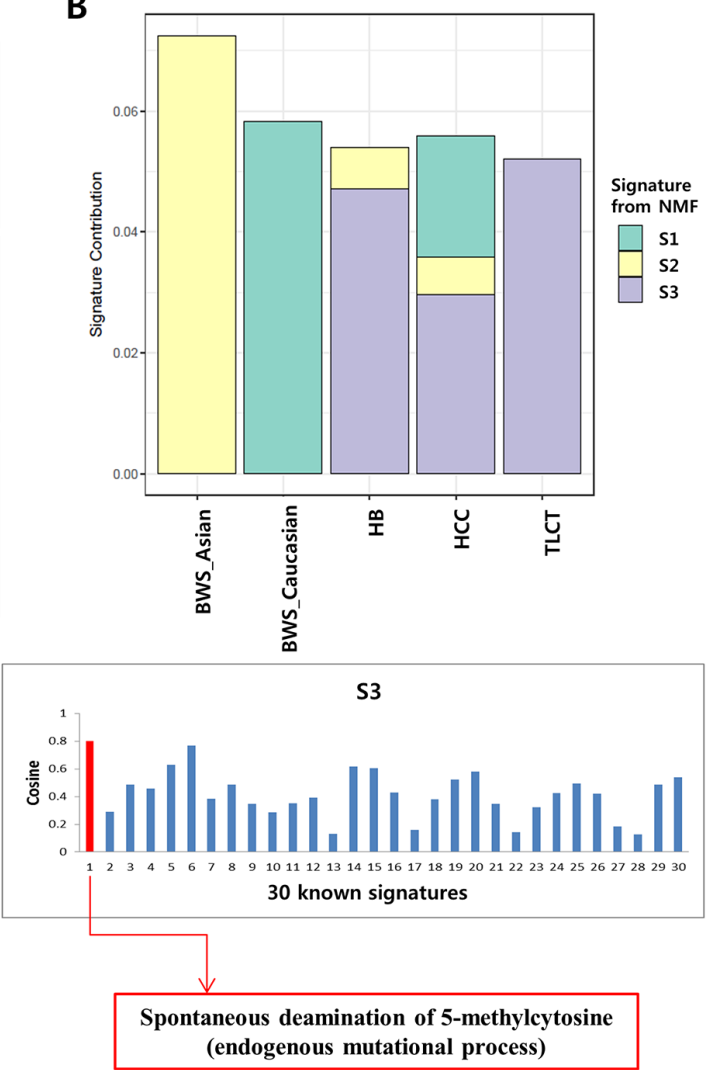

Figure 5: Mutation signatures of hepatoblastomas. (A) Mutation spectra based on 96 base substitutions for the three signatures generated from NMF algorithm. (B) Proportion of the total substitutions contributed by each of the three mutational signatures (S1, BWS (Caucasian); S2, BWS (this case); S3, other liver tumors). (C) The graph shows similarities between three mutational signatures and 30 known mutation signatures from the COSMIC database. Red bars indicate high similarities with cosine similarity $\geq 0.8$. 
and PALB2 assessed by both SIFT and MutationTaster were "tolerable" (Supplementary Table 3). There was no $C D K N 1 C$ germline mutation known to be mutated in about $5-10 \%$ of sporadic BWS and in about $50 \%$ of familial BWS [18].

\section{DISCUSSION}

The aim of this study was three-fold. First, we aimed to disclose somatic mutations in entire coding genes of a hepatoblastoma in a BWS patient. Second, we attempted to analyze genome-wide CNA and $\mathrm{LOH}$ profiles in that patient. Third, we attempted to find genomic alterations predisposing to BWS-related hepatoblastoma development besides chromosome 11 p15.5 alterations. In a hepatoblastoma arising from this BWS infant, we report hitherto unknown germline and somatic genomic alterations. At somatic levels, previously known CTNNB1 mutation and chromosome lq gain were identified driver events, but no additional driver events were uncovered in the hepatoblastoma. In a previous WES of a hepatoblastoma with BWS, there was only a CTNNB1 mutation in the tumor [9]. Together, it appears that there may not be additional somatic alterations in BWSrelated hepatoblastoma compared to those in sporadic hepatoblastomas. At germline levels, we confirmed the genetic cause of BWS in the patient by identifying $11 \mathrm{p} 15.5$ UPD. In addition, we detected $A P C$ and $P A L B 2$ missense germline mutations. PALB2 interacts and cooperates with BRCA2, and mediates DNA damage repair. PALB2 is a tumor suppressor gene; its alteration of which was known to predispose to childhood Wilms' tumor and medulloblastoma as well as breast cancers [19]. Of note, the PALB2 germline mutation (p.E837K) identified in our study was previously reported in familial breast cancer [20]. Many reports have found the presence of $A P C$ germline mutations associated with hepatoblastoma especially in the kindreds of FAP [21, 22]. Even in patients with apparently sporadic hepatoblastomas, germline $A P C$ mutations are identified in about $10 \%$ [8]. Although the $A P C$ and $P A L B 2$ germline mutations were not predicted as being pathogenic, further studies are necessary to conclude whether these two mutations play a synergistic role for the tumorigenesis of hepatoblastoma.

Analyses of the mutation signatures of our and a previous case [9] of hepatoblastomas with BWS showed that their signatures were distinct from sporadic hepatoblastomas. It is possible that the difference could come from the difference between genetic backgrounds (germline (BWS) and sporadic). However, the sample size was too small to conclude the hypothesis and further studies with a larger cohort of hepatoblastoma with BWS should be performed to clarify our observation. Children with BWS are at increased risk of tumors, therefore tumor surveillance particularly for Wilms' tumor and hepatoblastoma is recommended for them [12]. We tried to follow the recommended tumor surveillance protocol of AFP and abdominal ultrasonography every 2-3 months for this patient. AFP level was checked according to the schedule, while ultrasonography was not, because the parents did not follow the instructions properly, which resulted in the delay of diagnosis of hepatoblastoma by two months. Our data strongly suggest that it is very important to follow the surveillance protocol strictly and to inform the parents of the possible consequences of the delay. Current data on the best schedule for the tumor surveillance are limited (2-3 months schedule interval for most recommendations) [23]. Several studies suggest the use of more intense protocols that adopt shortened intervals in early infancy, especially in babies with UPD or severe phenotypes [24, 25]. Even though the patient did not show severe phenotype of BWS, based on the genetic analysis, we can conclude that this baby was a high-risk patient and that strict and intense surveillance protocol (even shorter than 3 months) would have made the earlier diagnosis possible.

In summary, we found chromosome $11 \mathrm{p} 15.5$ UPD as a cause of BWS as well as germline mutations of $A P C$ and $P A L B 2$ genes in a hepatoblastoma patient. Germline concurrence of 11p15.5 UDP, $A P C$ and PALB2 in a hepatoblastoma is reported for the first time. As for somatic alterations, we detected a CTNNB1 hotspot mutation and chromosome 1q gain, both of which were well-known somatic alterations in hepatoblastoma, suggesting a pivotal role for development of the hepatoblastoma. To our knowledge, this is the first report of germline and somatic genomic alteration profiles in hepatoblastoma arising from BWS. The development of hepatoblastoma in this BWS infant can be explained by predisposition of UPD at $11 \mathrm{p} 15.5$ as well as possibly of $A P C$ and $P A L B 2$ mutations and later by somatic events with $C T N N B 1$ somatic mutation and $1 \mathrm{q}$ gain acting as driver alterations. Clinically, our study provides a rationale for performing a more strict and intense protocol for hepatoblastoma surveillance in the UPD-carrying BWS infant.

\section{MATERIALS AND METHODS}

\section{Tissue sampling}

Formalin-fixed paraffin-embedded (FFPE) tumor tissue of the hepatoblastoma was obtained from Seoul St. Mary's Hospital of the Catholic University (Seoul, Korea). Informed consent from the parents and approval for this study were obtained from the Institutional Review Board of the Catholic University of Korea, College of Medicine. FFPE tumor tissue and adjacent normal liver tissue (hepatitis (-) and cirrhosis (-)) were manually microdissected by a pathologist. Genomic DNA was prepared using GeneRead DNA FFPE Kit (Qiagen, Hilden, Germany). 


\section{Whole-exome sequencing and variant calling}

WES analyses for both tumor and normal genomic DNA were performed using the Agilent SureSelect Human All Exome 50Mb Kit (Agilent Technologies, Santa Clara, CA) and Illumina HiSeq2000 platform according to the manufacturer's instructions. $101 \mathrm{bp}$ paired-end reads were generated using the Illumina HiSeq2000 platform and the Burrows-Wheeler aligner was used to align the sequencing reads onto the human reference genome (hg19). Somatic variants were identified using MuTect [26] and SomaticIndelDetector [27] for point mutations and indels, respectively. Germline variants were identified using GATK HaplotypeCaller. All variants were annotated using ANNOVAR [28]. Mutation rates and spectra were compared with previously reported studies of hepatoblastoma [10,11], transient liver cell tumor (TLCT) [10], and adult hepatocellular carcinoma (HCC) from The Cancer Genome Atlas (TCGA).

\section{DNA copy number and loss of heterozygosity analyses}

Single nucleotide polymorphism (SNP) array analysis for both normal and tumor genomic DNA was performed using OncoScan FFPE Assay Kit (Affymetrix, Santa Clara, CA). The DNA copy number and loss of heterozygosity ( $\mathrm{LOH})$ were analyzed and visualized using the SNP-FASST2 Segmentation statistical algorithm in NEXUS software v7.5 (Biodiscovery Inc., El Segundo, $\mathrm{CA}$ ). All of the identified CNAs and $\mathrm{LOH}$ events were manually curated in terms of $\log _{2}$ ratio and $\mathrm{B}$ allele frequency.

\section{Mutational signature analysis}

Mutational signature analyses were conducted using SomaticSignatures [29] $\mathrm{R}$ package which estimates somatic signatures with a non-negative matrix factorization algorithm. We analyzed mutational signatures of hepatoblastomas including our BWS patient, previously reported hepatoblastoma [10,11], TLCT [10], adult HCC from The Cancer Genome Atlas (TCGA), and a recently reported Caucasian BWS patient [9]. Similarities between mutational signatures were calculated using a cosine correlation similarity method with 30 known signatures recorded in the Catalogue of Somatic Mutations in Cancer (COSMIC) database [30].

\section{Author contributions}

YJC and SugHL conceived of the study. SYK, SHJ, YJC and SugHL wrote the article. SYK, SHJ and MSK performed experiments. MRH, SYK and HCP performed computation analyses. ESJ, SHL and SugHL collected the specimen and performed clinical review. All authors have read and approved the manuscript for publication.

\section{CONFLICTS OF INTEREST}

None declared.

\section{FUNDING}

This study was supported by a grant from National Research Foundation of Korea (2012R1A5A2047939 and NRF-2015M3C9A4053389) and by a grant from Korea Healthcare Technology R\&D Project (HI14C3417).

\section{REFERENCES}

1. Weksberg R, Shuman C, Beckwith JB. BeckwithWiedemann syndrome. Eur J Hum Genet. 2010; 18:8-14.

2. Cooper WN, Luharia A, Evans GA, Raza H, Haire AC, Grundy R, Bowdin SC, Riccio A, Sebastio G, Bliek J, Schofield PN, Reik W, Macdonald F, et al. Molecular subtypes and phenotypic expression of BeckwithWiedemann syndrome. Eur J Hum Genet. 2005; 13:1025-1032.

3. Henry I, Puech A, Riesewijk A, Ahnine L, Mannens M, Beldjord C, Bitoun P, Tournade MF, Landrieu P, Junien C. Somatic mosaicism for partial paternal isodisomy in Wiedemann-Beckwith syndrome: a post-fertilization event. Eur J Hum Genet. 1993; 1:19-29.

4. DeBaun MR, Tucker MA. Risk of cancer during the first four years of life in children from The BeckwithWiedemann Syndrome Registry. J Pediatr. 1998; 132:398-400.

5. Rump P, Zeegers MP, van Essen AJ. Tumor risk in Beckwith-Wiedemann syndrome: A review and metaanalysis. Am J Med Genet A. 2005; 136:95-104.

6. Spector LG, Birch J. The epidemiology of hepatoblastoma. Pediatr Blood Cancer. 2012; 59:776-779.

7. Tomlinson GE, Kappler R. Genetics and epigenetics of hepatoblastoma. Pediatr Blood Cancer. 2012; 59:785-792.

8. Aretz S, Koch A, Uhlhaas S, Friedl W, Propping P, von Schweinitz D, Pietsch T. Should children at risk for familial adenomatous polyposis be screened for hepatoblastoma and children with apparently sporadic hepatoblastoma be screened for APC germline mutations? Pediatr Blood Cancer. 2006; 47:811-818.

9. Sumazin P, Chen Y, Trevino LR, Sarabia SF, Hampton OA, Patel K, Mistretta TA, Zorman B, Thompson P, Heczey A, Comerford S, Wheeler DA, Chintagumpala M, et al. Genomic analysis of hepatoblastoma identifies distinct molecular and prognostic subgroups. Hepatology. 2017; 65:104-121.

10. Eichenmuller M, Trippel F, Kreuder M, Beck A, Schwarzmayr T, Haberle B, Cairo S, Leuschner I, von Schweinitz D, Strom TM, Kappler R. The genomic landscape of hepatoblastoma and their progenies with HCClike features. J Hepatol. 2014; 61:1312-1320. 
11. Jia D, Dong R, Jing Y, Xu D, Wang Q, Chen L, Li Q, Huang Y, Zhang Y, Zhang Z, Liu L, Zheng S, Xia Q, et al. Exome sequencing of hepatoblastoma reveals novel mutations and cancer genes in the Wnt pathway and ubiquitin ligase complex. Hepatology. 2014; 60:1686-1696.

12. Tan TY, Amor DJ. Tumour surveillance in BeckwithWiedemann syndrome and hemihyperplasia: a critical review of the evidence and suggested guidelines for local practice. J Paediatr Child Health. 2006; 42:486-490.

13. Totoki Y, Tatsuno K, Yamamoto S, Arai Y, Hosoda F, Ishikawa S, Tsutsumi S, Sonoda K, Totsuka H, Shirakihara T, Sakamoto H, Wang L, Ojima H, et al. High-resolution characterization of a hepatocellular carcinoma genome. Nat Genet. 2011; 43:464-469.

14. Koch A, Denkhaus D, Albrecht S, Leuschner I, von Schweinitz D, Pietsch T. Childhood hepatoblastomas frequently carry a mutated degradation targeting box of the beta-catenin gene. Cancer Res. 1999; 59:269-273.

15. Polakis P. Wnt signaling and cancer. Genes Dev. 2000; 14:1837-1851.

16. Huang da W, Sherman BT, Lempicki RA. Systematic and integrative analysis of large gene lists using DAVID bioinformatics resources. Nat Protoc. 2009; 4:44-57.

17. Park JY, Zhang F, Andreassen PR. PALB2: the hub of a network of tumor suppressors involved in DNA damage responses. Biochim Biophys Acta. 2014; 1846:263-275.

18. Lam WW, Hatada I, Ohishi S, Mukai T, Joyce JA, Cole TR, Donnai D, Reik W, Schofield PN, Maher ER. Analysis of germline CDKN1C (p57KIP2) mutations in familial and sporadic Beckwith-Wiedemann syndrome (BWS) provides a novel genotype-phenotype correlation. J Med Genet. 1999; 36:518-523.

19. Reid S, Schindler D, Hanenberg H, Barker K, Hanks S, Kalb R, Neveling K, Kelly P, Seal S, Freund M, Wurm M, Batish SD, Lach FP, et al. Biallelic mutations in PALB2 cause Fanconi anemia subtype FA-N and predispose to childhood cancer. Nat Genet. 2007; 39:162-164.

20. Kim H, Cho DY, Choi DH, Oh M, Shin I, Park W, Huh SJ, Nam SJ, Lee JE, Kim SW. Frequency of pathogenic germline mutation in CHEK2, PALB2, MRE11, and RAD50 in patients at high risk for hereditary breast cancer. Breast Cancer Res Treat. 2017; 161:95-102.

21. Oda H, Imai Y, Nakatsuru Y, Hata J, Ishikawa T. Somatic mutations of the APC gene in sporadic hepatoblastomas. Cancer Res. 1996; 56:3320-3323.
22. Hirschman BA, Pollock BH, Tomlinson GE. The spectrum of APC mutations in children with hepatoblastoma from familial adenomatous polyposis kindreds. J Pediatr. 2005; 147:263-266.

23. Mussa A, Di Candia S, Russo S, Catania S, De Pellegrin M, Di Luzio L, Ferrari M, Tortora C, Meazzini MC, Brusati R, Milani D, Zampino G, Montirosso R, et al. Recommendations of the Scientific Committee of the Italian Beckwith-Wiedemann Syndrome Association on the diagnosis, management and follow-up of the syndrome. Eur J Med Genet. 2016; 59:52-64.

24. Smith AC, Shuman C, Chitayat D, Steele L, Ray PN, Bourgeois J, Weksberg R. Severe presentation of BeckwithWiedemann syndrome associated with high levels of constitutional paternal uniparental disomy for chromosome 11p15. Am J Med Genet A. 2007; 143A:3010-3015.

25. Mussa A, Ferrero GB, Ceoloni B, Basso E, Chiesa N, De Crescenzo A, Pepe E, Silengo M, de Sanctis L. Neonatal hepatoblastoma in a newborn with severe phenotype of Beckwith-Wiedemann syndrome. Eur J Pediatr. 2011; 170:1407-1411.

26. Cibulskis K, Lawrence MS, Carter SL, Sivachenko A, Jaffe D, Sougnez C, Gabriel S, Meyerson M, Lander ES, Getz G. Sensitive detection of somatic point mutations in impure and heterogeneous cancer samples. Nat Biotechnol. 2013; 31:213-219.

27. DePristo MA, Banks E, Poplin R, Garimella KV, Maguire JR, Hartl C, Philippakis AA, del Angel G, Rivas MA, Hanna M, McKenna A, Fennell TJ, Kernytsky AM, et al. A framework for variation discovery and genotyping using next-generation DNA sequencing data. Nat Genet. 2011; 43:491-498.

28. Wang K, Li M, Hakonarson H. ANNOVAR: functional annotation of genetic variants from high-throughput sequencing data. Nucleic Acids Res. 2010; 38:e164.

29. Gehring JS, Fischer B, Lawrence M, Huber W. SomaticSignatures: inferring mutational signatures from single-nucleotide variants. Bioinformatics. 2015; 31:3673-3675.

30. Forbes SA, Beare D, Gunasekaran P, Leung K, Bindal N, Boutselakis H, Ding M, Bamford S, Cole C, Ward S, Kok CY, Jia M, De T, et al. COSMIC: exploring the world's knowledge of somatic mutations in human cancer. Nucleic Acids Res. 2015; 43:D805-811. 